\section{References}

1. Modi SR, Collins JJ, Relman DA. Antibiotics and the gut microbiota J Clin Invest. 2014; 124(10):4212-4218.

2. Quraishi MN, Widlak M, Bhala N et al. Systematic review with meta-analysis: the efficacy of faecal microbiota transplantation for the treatment of recurrent and refractory Clostridium difficile infection. Aliment Pharmacol Ther. 2017;46(5):479-493.

3. Saha S, Tariq R, Tosh PK, et al. Faecal microbiota transplantation for eradicating carriage of multidrug-resistant organisms: a systematic review. Clin Microbiol Infect. 2019;25(8):958-963.

4. Bilinski J, Grzesiowski P, Sorensen N, et al. Fecal microbiota transplantation in patients with blood disorders inhibits gut colonization with antibiotic-resistant bacteria: results of a prospective, single-center study. Clin Infect Dis. 2017;65(3):364-370.

5. Galloway-Pena J, Brumlow C, Shelburne S. Impact of the microbiota on bacterial infections during cancer treatment. Trends Microbiol. 2017;25(12):992-1004

6. Montassier E, Gastinne T, Vangay P, et al. Chemotherapy-driven dysbiosis in the intestinal microbiome. Aliment Pharmacol Ther. 2015;42(5):515-528.

7. Shogbesan O, Poudel DR, Victor S, et al. A systematic review of the efficacy and safety of fecal microbiota transplant for Clostridium difficile infection in immunocompromised patients. Can J Gastroenterol Hepatol. 2018;2018:1394379.

8. Wang S, Xu M, Wang W, et al. Systematic review: adverse events of fecal microbiota transplantation. PLoS One. 2016;11(8):e0161174.

9. Shono Y, van den Brink MRM. Gut microbiota injury in allogeneic haematopoietic stem cell transplantation. Nat Rev Cancer.
2018;18(5):283-295.

10. Battipaglia G, Malard F, Rubio MT, et al. Fecal microbiota transplantation before or after allogeneic hematopoietic transplantation in patients with hematologic malignancies carrying multidrug-resistance bacteria. Haematologica 2019;104(8):1682-1688.

11. Stalenhoef JE, Terveer EM, Knetsch CW, et al. Fecal microbiota transfer for multidrug-resistant gram-negatives: a clinical success combined with microbiological failure. Open Forum Infect Dis 2017;4(2): ofx047.

12. Kelly CR, Kahn S, Kashyap P, et al. Update on fecal microbiota transplantation 2015: indications, methodologies, mechanisms, and outlook. Gastroenterology. 2015;149(1):223-237.

13. Khoruts A, Sadowsky MJ. Understanding the mechanisms of faecal microbiota transplantation. Nat Rev Gastroenterol Hepatol. 2016;13(9):508-516.

14. O'Neill J. Review on antimicrobial resistance antimicrobial resistance: tackling a crisis for the health and wealth of nations. Review on Antimicrobial Resistance. London, 2014. Available from: http://amr-review.org

15. Abu-Sbeih H, Ali FS, Wang Y. Clinical review on the utility of fecal microbiota transplantation in immunocompromised patients. Curr Gastroenterol Rep. 2019;21(4):8

16. Wardill HR, Secombe KR, Bryant RV, et al. Adjunctive fecal microbiota transplantation in supportive oncology: Emerging indications and considerations in immunocompromised patients. EBioMedicine. 2019;44 730-740.

17. DeFilipp Z, Hohmann E, Jenq RR, et al. Fecal microbiota transplantation: restoring the injured microbiome after allogeneic hematopoietic cell transplantation. Biol Blood Marrow Transplant. 2019;25(1) e17-e22.

\title{
Predicting risk for recurrence of arterial ischemic stroke in children: thrombophilia as another piece of the puzzle
}

\section{Ghada Aborkhees and Lesley Gayle Mitchell}

Department of Pediatrics, University of Alberta, Edmonton, AB, Canada

E-mail: LESLEY GAYLE MITCHELL - Lesley.Mitchell@albertahealthservices.ca

doi:10.3324/haematol.2019.222695

$\mathrm{R}$ ecurrent arterial ischemic stroke (AIS) is increasingly recognized as a significant cause of mortality and morbidity in the pediatric population. Identifying risk factors for recurrent AIS is essential for developing strategies for secondary stroke prevention. While multiple risk factors have been identified for AIS events, the only confirmed risk factor for initial AIS recurrence is the presence of vasculopathy, particularly moyamoya disease. ${ }^{1-3}$ In a meta-analysis, prothrombotic risk factors were found to be associated with AIS in pediatric patients. ${ }^{4}$ However, the role of thrombophilia as an independent risk factor for recurrent AIS has not been established due to a paucity of research in the area and the lack of statistical power in the published studies.

In the current edition of Haematologica, deVeber et al. report on an international prospective cohort study which recruited 894 pediatric patients from centers in Germany, Canada and the UK. ${ }^{5}$ The primary objective of the study was to determine the association of prothrombotic risk factors and/or underlying stroke subtypes with risk for recurrent stroke. The authors excluded asymptomatic strokes and transient ischemic attacks due to the difference in underlying disease as well as the differing outcomes from symptomatic strokes. Sickle cell disease and moyamoya vasculopathies were also excluded as their recurrence rates and risk factors differ from those of other subtypes of pediatric AIS. The authors report an overall AIS recurrence rate of $17.9 \%$ in the cohort studied. The study confirmed the association of vasculopathy as a risk factor for AIS recurrence. The novel approach in the current study was the examination of the role of thrombophilia as an independent risk factor for AIS recurrence. Study patients were excluded if they had thrombophilic markers with established pathophysiological relevance such as homozygous protein $\mathrm{C}$ and homozygous antithrombin deficiency. Analysis of the study data showed that the following were independent risk factors for recurrence: antithrombin deficiency (hazard ratio 3.9; $95 \%$ confidence interval: 1.4-10.9), increased lipoprotein(a) (hazard ratio 2.3; 95\% confidence interval: 1.3-4.1) and more than one prothrombotic marker (hazard ratio 1.9; $95 \%$ confidence interval: $1.1-3.2)$. The results obtained from this study highlight the importance of screening AIS cases for thrombophilia in order to identify the children at risk of AIS recurrence.

The reported study is a valuable addition to the previous efforts to identify the risk factors for AIS recurrence. There are significant strengths of the study design. The first was the relatively large sample size, which provided adequate power to determine the association of pro- 
thrombotic markers with recurrence. Second, recruiting patients by collaboration of investigators from three countries and including multiple sites supports the generalizability of the results. As the study sites were large tertiary or quaternary centers, another key element of the design was the exclusion of referrals from outside the catchment area, which minimizes referral bias. One study showed that there is a higher rate of AIS recurrence among referrals, providing evidence of differences in the populations of patients which would affect generalizability of the results. ${ }^{3}$ Furthermore, prothrombotic marker testing was performed at each site, which demonstrates the ability to determine these markers in different clinical laboratories. Finally, there was a clear clinical and radiologically combined definition of initial AIS and recurrent stroke events.

There are a few limitations to the study. The first is combining all three clinical entities for determination of association of prothrombotic markers with recurrence risk. While this could be considered a strength, as the results are generalizable to the pediatric population with AIS, there is a missed opportunity to determine markers specific to each clinical population. As the mechanisms for AIS and AIS recurrence likely differ based on the underlying disorder, it is reasonable to predict that the markers will vary by diagnosis. Another important limitation is the extended study period from 1990 to 2016. While this allowed the enrollment of a large number of patients with incident AIS, over time there are variations in clinical practice including index case diagnosis and interventions. These factors could influence patients' outcomes as well as characterization of the study populations, which affects the generalizability of the study results. However, even with the limitations, the results of the study are valid and are a valuable contribution to this area of research.

In pediatric AIS studies international collaboration is essential to assemble adequately powered cohorts for determination of predictive markers for AIS recurrence. In futures studies, with recent publications in the area including the current paper, there are now avenues for determin- ing predictive models which include clinical variables and biomarkers. For example, the Childhood AIS Standardized Classification and Diagnostic Evaluation (CASCADE) classification is a consensus-based standardized tool for classifying arteriopathic and non-arteriopathic AIS. Patients with arteriopathy classified as CASCADE 2 (unilateral focal cerebral arteriopathy) and 3 (bilateral cerebral arteriopathy) have an increased risk of AIS recurrence as do those classified as CASCADE 5 (patients with cardio-embolism). ${ }^{6} \mathrm{~A}$ recent publication by Fullerton et al. identified inflammatory markers associated with risk of recurrence in arteriopathic patients. Therefore, a model could be based on a combination of clinical variables and both thrombophilia and inflammatory markers. The current publication by deVeber et al. will help to shape future studies determining predictive models. These predictive models will allow secondary prophylaxis interventions to be targeted to only those children at risk of recurrence, ultimately improving the care of children who have had an AIS.

\section{References}

1. Strater R, Becker S, von EA, et al. Prospective assessment of risk factors for recurrent stroke during childhood--a 5-year follow-up study. Lancet. 2002;360(9345):1540-1545.

2. Ganesan V, Prengler M, Wade A, Kirkham FJ. Clinical and radiological recurrence after childhood arterial ischemic stroke. Circulation. 2006;114(20):2170-2177.

3. Stacey A, Toolis C, Ganesan V. Rates and risk fctors for arterial ischemic stroke recurrence in children. Stroke. 2018;49(4):842-847.

4. Kenet G, Lutkhoff LK, Albisetti M, et al. Impact of thrombophilia on risk of arterial ischemic stroke or cerebral sinovenous thrombosis in neonates and children: a systematic review and meta-analysis of observational studies. Circulation. 2010;121(16):1838-1847.

5. deVeber G, Kirkham F, Shannon K, et al. Recurrent stroke: the role of thrombophilia in a large international pediatric stroke population. Haematologica. 2019;104(8):1676-1681.

6. Bohmer M, Niederstadt T, Heindel W, et al. Impact of childhood arterial ischemic stroke standardized classification and diagnostic evaluation classification on further course of arteriopathy and recurrence of childhood stroke. Stroke. 2018 Dec 7. [Epub ahead of print]

7. Fullerton HJ, deVeber GA, Hills NK, et al. Inflammatory biomarkers in childhood arterial ischemic stroke: correlates of stroke cause and recurrence. Stroke. 2016;47(9):2221-2228. 